\title{
The $\widetilde{C} \rightarrow \widetilde{A}$ emission in water: Theory and experiment
}

\author{
V. Engel, G. Meijer, ${ }^{a)}$ A. Bath, P. Andresen, and R. Schinke \\ Max-Planck-Institut für Strömungsforschung, 3400 Göttingen, Federal Republic of Germany
}

(Received 6 March 1987; accepted 8 July 1987)

The $\widetilde{C} \rightarrow \widetilde{A}$ emission spectra for $\mathrm{H}_{2} \mathrm{O}$ and $\mathrm{D}_{2} \mathrm{O}$ are measured and calculated. The theoretical model is based on an exact treatment of the dissociation dynamics of the $\widetilde{A}$ state using a calculated potential energy surface. Agreement with the measurements is excellent. The spectra extend from $\lambda \sim 380 \mathrm{~nm}$ up to $\lambda \sim 600 \mathrm{~nm}$ with maxima around $425\left(\mathrm{H}_{2} \mathrm{O}\right)$ and 440 $\mathrm{nm}\left(\mathrm{D}_{2} \mathrm{O}\right)$.

\section{INTRODUCTION}

The photodissociation of water with excitation energies smaller than $10 \mathrm{eV}$ has been the subject of many experimen$\mathrm{tal}^{1-9}$ and theoretical ${ }^{10-14}$ investigations over the last few decades. This list of references is by far incomplete. Now there exists a reasonable accord concerning the electronic identity of most of the dominant features ${ }^{15}$ and the more general aspects of the dissociation dynamics are well understood.

Figure 1 shows, highly schematically, the four lowest electronic states of water which are involved in the photodissociation at energies below $10 \mathrm{eV}$. Plotted are the corresponding potential energy curves vs the $\mathrm{H}-\mathrm{OH}$ separation, $\boldsymbol{R}_{\mathrm{H}-\mathrm{OH}}$ for fixed internal $\mathrm{OH}$ stretch coordinate, $\boldsymbol{r}_{\mathrm{OH}}$ and fixed bending angle, $\alpha$. For a more complete representation see, for example, Refs. 12 and 16. According to Fig. 1 we distinguish between three different dissociation processes which are summarized in Fig. 2 and briefly discussed below.

(1) At photon wavelengths larger than $\sim 150 \mathrm{~nm}$ only the first excited state, $\widetilde{A}^{1} B_{1}$ can be reached. The corresponding potential is steeply repulsive in the $\boldsymbol{R}_{\mathrm{H}-\mathrm{OH}}$ coordinate leading to fast and direct dissociation and a broad, more or less structureless absorption continuum with a maximum around $\lambda \sim 165 \mathrm{~nm} .^{1,5}$ The $\mathrm{OH}$ products are in the electronic ground state, rotationally cold but with a significant degree of vibrational excitation. ${ }^{8,17,18}$ Since the $\widetilde{A}$ state is well separated from any other state process (1) is an ideal case of direct photodissociation and well suited for a rigorous theoretical treatment. Almost exact dynamical calculations have been performed on the basis of a calculated, three-dimensional potential energy surface ${ }^{19}$ and astonishing agreement with all available experimental data is achieved. ${ }^{14,18,20} \mathrm{We}$ can now safely claim that process (1) is fully understood. ${ }^{21}$

(2) At wavelengths below $\sim 150 \mathrm{~nm}$ absorption and dissociation proceed via the $\widetilde{B}^{1} A_{1}$ state. The corresponding potential energy surface is much more complex than for the $\widetilde{A}$ state. It is generally less repulsive and has a deep well for bending angles around $180^{\circ}$ due to an avoided crossing with the ground state $\widetilde{X}$. The trajectories which evolve entirely on the $\widetilde{B}$ surface, process 2 (a), lead to electronically excited $\mathrm{OH}$ molecules. Resolving the $A \rightarrow X$ emission of $\mathrm{OH}$ one ob-

\footnotetext{
a) Permanent address: Universiteit Nijmegen, Nijmegen, The Netherlands.
}

tains the corresponding rotational-vibrational distribution. $3,7,22,23$ The rotational distribution is highly inverted and peaks very close to the highest accessible state. A dynamical study of process 2(a) has been performed by Segev and Shapiro ${ }^{13,24}$ using the potential surface of Flouquet and Horsley. ${ }^{10}$ However, a direct comparison with the experimental absorption cross section and the rotational distribution has not been reported.

The quantum yield for electronically excited $\mathrm{OH}(A)$ is less than $10 \% .{ }^{25}$ Due to nonadiabatic coupling, ${ }^{26}$ more than $90 \%$ of the molecules dissociate either along the $\tilde{A}$ state, process 2 (b), or via the $\widetilde{X}$ state, process $2(\mathrm{c})$. In both cases the $\mathrm{OH}$ products are in the electronic ground state. The correct dissociation pathway is not really known up to now. Unfortunately, reliable dynamical calculations including nonadiabatic effects, will not be possible for the near future. Recently measured internal energy distributions of the ground state $\mathrm{OH}(X)$ products may be helpful to decide whether process $2(\mathrm{~b})$ or 2 (c) is the important channel. ${ }^{9}$

(3) Also at wavelengths below $\sim 125 \mathrm{~nm}$, direct excita-

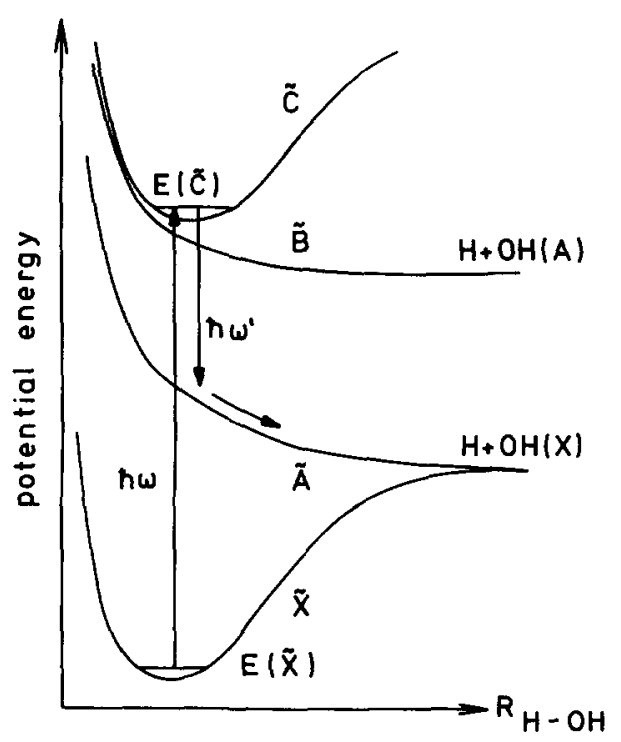

FIG. 1. Schematic representation of the four lowest states of water which are involved in the photodissociation at energies below $10 \mathrm{eV}$. Shown are the potential curves vs $\mathrm{H}-\mathrm{OH}$ separation for fixed intramolecular $\mathrm{OH}$ coordinate and fixed bending angle. 
1) $\lambda \geq 150 \mathrm{~nm}$

$\mathrm{H}_{2} \mathrm{O}(\tilde{X})+\mathrm{H \omega} \longrightarrow \mathrm{H}_{2} \mathrm{O}(\tilde{\mathrm{A}}) \longrightarrow \mathrm{H}+\mathrm{OH}(X)$

2) $\lambda \leqslant 150 \mathrm{~nm}$

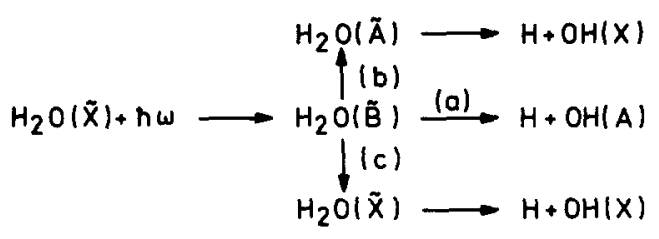

3) $\lambda \leqslant 125 \mathrm{~nm}$

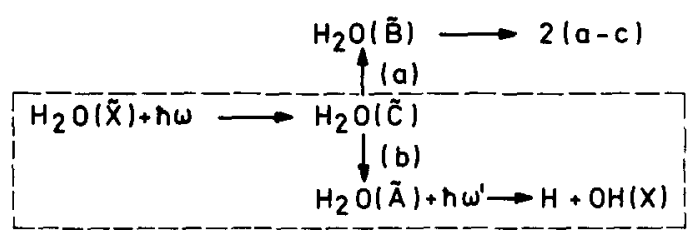

FIG. 2. Summary of the possible dissociation pathways for water.

tion into the $\widetilde{C}^{1} B_{1}$ state is possible and has been resolved. ${ }^{4,6}$ The $\widetilde{C}$ state is strongly coupled to the $\widetilde{B}$ state which leads to fast predissociation, process 3 (a). However, the observation of resolvable rotational structure implies predissociation lifetimes at least in the picosecond range. This opens an alternative channel, the bound-free $\widetilde{C} \rightarrow \widetilde{A}$ spontaneous emission, process $3(b)$, followed by a fast and direct dissociation via the repulsive $\tilde{A}$ state. The final steps of process (1) and process 3 (b) are identical. The lifetime within the $\widetilde{C}$ state is evidently long enough to allow the observation of this weak emission at $\lambda \sim 420 \mathrm{~nm} .{ }^{27}$ As stated by Docker et al..$^{27}$ this is "the first assigned bound to free emission observed in a polyatomic molecule."

The bound to free emission from the $\widetilde{C}$ to the $\tilde{A}$ state is the topic of this article. The $\widetilde{A}$ state potential is known from the work of Staemmler and Palma ${ }^{19}$ and the dissociation dynamics in the $\tilde{A}$ state can be treated almost exactly. ${ }^{14,20}$ The astonishing success to treat the $\widetilde{X} \rightarrow \widetilde{A}$ absorption in all details proves to us that both ingredients are highly reliable. Therefore, it is most appealing to calculate the $\widetilde{C} \rightarrow \widetilde{A}$ emission spectrum on a dynamical basis.

In Sec. II we will very briefly summarize the basic aspects of the calculation. The bound free emission spectra of $\mathrm{H}_{2} \mathrm{O}$ and $\mathrm{D}_{2} \mathrm{O}$ have been measured over an extended wavelength range with very good $S / N$. The results are in rough qualitative, but not in quantitative agreement with the measurements of Docker et al. ${ }^{27}$ The experiments are described in Sec. III. The theoretical and experimental results for $\mathrm{H}_{2} \mathrm{O}$ and $\mathrm{D}_{2} \mathrm{O}$ are compared and discussed in Sec. IV. The conclusions of this study are summarized in Sec. V.

\section{THEORY}

The cross section for emission of a photon with energy $\omega$ is calculated in the time-independent treatment by the usual Golden Rule expression which follows from first-order perturbation theory for the light-matter interaction. ${ }^{28}$ In the time-independent formulation one first calculates the partial cross sections for emitting a photon $\omega$ and producing $\mathrm{OH}$ in a particular vibrational-rotational state $(n j)$. The total emission cross section is then the sum over all partial cross sections.

As usual, the partial cross section is calculated from ${ }^{28}$

$$
\sigma_{n j}(\omega) \sim \omega^{3}\left|\left\langle\Psi_{b}^{(i)}\left|\mu_{\epsilon}\right| \Psi_{d}^{(-n /)}\right\rangle\right|^{2},
$$

where $\Psi_{b}^{(i)}$ is the bound $(b)$ state nuclear wave function in a specific initial state ( $i)$ and $\Psi_{d}^{(-n j)}$ is the nuclear wave function in the dissociation $(d)$ state. The superscript $(-n j)$ denotes the dissociation into a specific $\mathrm{OH}$ vibrational-rotational state..$^{28}$ Equation (1) is identical for emission and absorption, except for the $\omega^{3}$ prefactor which is appropriate for spontaneous emission. The electronic degree of freedom ( $\Lambda$ doublets) is ignored in this study. $\mu_{\epsilon}$ is the component of the transition dipole function in the direction of the polarization of the electric vector. All quantities in Eqs. (1) are three dimensional.

The main problem in applying Eq. (1) is the calculation of the free, dissociative wave function $\Psi_{d}^{(-n j)}$, especially for a symmetric molecule like $\mathrm{H}_{2} \mathrm{O}$ which has two equivalent "reaction" channels. In our study of the $\widetilde{X} \rightarrow \tilde{A}$ dissociation ${ }^{14,20}$ we have solved this problem by introducing the energy sudden approximation (ESA) for the rotational degree of freedom. ${ }^{29}$ Within the ESA it is assumed that the rotational energy transfer during dissociation is small compared to the total available energy. This assumption effectively decouples the rotational (bending) degree of freedom from the two vibrational (stretching) degrees of freedom. The latter are then treated exactly by using two-dimensional polar coordinates. ${ }^{30}$

The ESA is highly accurate for the $\widetilde{X} \rightarrow \widetilde{A}$ dissociation of water because the $\widetilde{A}$ state potential surface is almost isotropic around the excitation region, i.e., the ground state equilibrium. ${ }^{31}$ Therefore, no appreciable torque is exerted on $\mathrm{OH}$ during dissociation and the final rotational distribution is almost completely determined by the wave function of the parent molecule. ${ }^{21}$ Since the equilibrium geometry of the $\widetilde{C}$ state is very close to that of the ground state ${ }^{4}$ the ESA is also appropriate to describe the $\widetilde{C} \rightarrow \widetilde{A}$ emission.

In Refs. 14 and 20 we used the empirical potential energy surface of Sorbie and Murrell ${ }^{32}$ to describe the $\widetilde{X}$ state. The nuclear wave function for the rotational-vibrational ground state was then calculated exactly. ${ }^{33}$ Unfortunately, an analytical expression for the $\widetilde{C}$ state potential energy surface is not available. Known are only (i) the equilibrium geometry, (ii) the rotational constants, (iii) the vibrational frequencies for the $(010)$ bending mode and the $(100)$ symmetric stretch mode, and (iv) the excitation energies. ${ }^{4,6}$ The molecular constants are all very similar to those of the ground state. For example, the equilibrium $\mathrm{OH}$ distance is $0.998 \AA$ instead of $0.957 \AA$ for $\widetilde{X}$ and the bending angle is $109.1^{\circ}$ instead of $104.5^{\circ}$. The vibrational frequencies differ only by $10 \%-20 \%$ from those of the ground state. Because of this similarity between $\widetilde{C}$ and $\widetilde{X}$ we did not attempt to construct a reasonable potential energy surface. We used directly the ground state nuclear wave function, however, shifted by $0.04 \AA$ to larger distances in order to account for 
the difference in equilibrium positions. With this choice of the bound nuclear wave function it is explicitly assumed that any distortion due to nonadiabatic coupling to the $\widetilde{B}$ state is absent.

In order to compare directly with the experimental spectrum on the wavelength axis it is necessary to fix the energetic position of the $\widetilde{C}$ state relative to the $\widetilde{A}$ state. This is unambiguously possible because the $\widetilde{X} \rightarrow \widetilde{C}$ excitation energy is accurately known from experiment. ${ }^{6}$ Describing the $\widetilde{X}$ and the $\tilde{A}$ states we used an energy normalization such that the three infinitely separated ground state atoms, $\mathbf{H}+\mathbf{H}+\mathbf{O}$, correspond to $E=0 .{ }^{20,32}$ With respect to this normalization the energies for the rotational-vibrational ground states are $E(\widetilde{X})=-9.500$ and $-9.655 \mathrm{eV}$ for $\mathrm{H}_{2} \mathrm{O}$ and $\mathrm{D}_{2} \mathrm{O}$, respectively, as calculated from the Sorbie and Murrell potential. The excitation energies are $80624.7\left(\mathrm{H}_{2} \mathrm{O}\right)$ and 80751.9 $\mathrm{cm}^{-1}\left(\mathrm{D}_{2} \mathrm{O}\right){ }^{6}$ This leads to $E(\widetilde{C})=0.496$ and $0.356 \mathrm{eV}$ as ground state levels within the $\widetilde{C}$ state for $\mathrm{H}_{2} \mathrm{O}$ and $\mathrm{D}_{2} \mathrm{O}$, respectively. We consider in the calculation only the emission from these levels to the $\widetilde{A}$ state assuming that the total angular momentum is zero for both states.

In the absence of any calculation we assume that the $\widetilde{C} \rightarrow \widetilde{A}$ transition dipole function is constant over the excitation region. The $\widetilde{X} \rightarrow \widetilde{A}$ transition moment indeed shows approximately such a behavior and it was demonstrated that the small coordinate dependence of the calculated moment has little effect on the absorption cross section. ${ }^{20}$ Nevertheless, this can be a rather limited restriction and our model for the $\widetilde{C} \rightarrow \widetilde{A}$ emission and the comparison of the calculated with the measured spectra must be judged in the light of this hypothesis.

Since the transition dipole function is not known it is impossible to calculate absolute cross sections. Therefore, the calculated quantities are all relative cross sections and only their wavelength dependence can be compared with experiment.

All details of the calculations will be extensively discussed in a forthcoming paper. ${ }^{20}$ It should be stressed that the calculations are parameter free.

\section{EXPERIMENT}

Because the experiments were done with the same setup that was described in detail in Ref. 34, only a brief outline is given. A tunable $\mathrm{KrF}$ laser is focused with a $100 \mathrm{~cm}$ lens into a $\mathrm{H}_{2} \mathrm{O}\left(\mathrm{D}_{2} \mathrm{O}\right)$ containing stainless steel vessel. A slow flow was maintained during the experiment and a typical operating pressure of $\sim 10$ Torr was used. The fluorescence was imaged with a 1:1 imaging optics to the entrance slit of a monochromator (McPherson 218). The entrance slit of the spectrometer together with the imaging optics determines the sampled fluorescence volume of $\sim 10 \mathrm{~mm}$ length and 1 $\mathrm{mm}$ diameter. The fluorescence was sampled from a region $\sim 4 \mathrm{~cm}$ away (upstream) from the focal point of the laser. No reasonable results were obtained from the focal point itself.

To reduce scattered light, some baffles are installed in the beam path of the KrF laser and also in the spectrometer. In some cases a filter was installed in the spectrometer to suppress direct $248 \mathrm{~nm}$ scatter of the laser. A window on top of the stainless steel vessel allowed to view the blue fluorescence. Although the excitation proceeds via a two photon process, the fluorescence was intense enough to be seen in a single laser pulse with the naked eye, if the $\mathrm{KrF}$ laser was tuned to a resonance. Considerably more fluorescence is observed for $\mathrm{D}_{2} \mathrm{O}$ than for $\mathrm{H}_{2} \mathrm{O}$, due to the longer predissociation lifetime of $\mathrm{D}_{2} \mathrm{O}$.

The dispersion spectra were recorded for excitation of different rotational levels in the $\widetilde{C}$ state. Although the present laser system has an improved locking efficiency, the emission spectra are independent of the prepared rotational state, in agreement with the results of Docker et al. ${ }^{27}$

The observed spectrum has been ascribed to the $\widetilde{C}-\tilde{A}$ emission in neutral $\mathrm{H}_{2} \mathrm{O}$ before. ${ }^{27}$ The present almost parameter-free calculations reproduce the spectrum almost quantitatively (see Sec. IV and Figs. 3 and 4) and this strongly supports this identification. Nevertheless, there has been some doubt, whether the emission originates from ionic $\mathrm{H}_{2} \mathrm{O}^{+}$instead from neutral $\mathrm{H}_{2} \mathrm{O}$. This doubt arises because $\mathrm{H}_{2} \mathrm{O}^{+}$has a spectrum in a similar wavelength range ${ }^{35}$ and secondly because the formation of $\mathrm{H}_{2} \mathrm{O}^{+}$is strongly enhanced whenever the laser is tuned into resonance with the $\widetilde{C}$ state. ${ }^{34}$ However, ionic emission can be ruled out by the following arguments. First, the $\mathrm{H}_{2} \mathrm{O}^{+}$spectrum from Ref. 35 is different. It peaks around $465 \mathrm{~nm}$ and shows pronounced structure, indicating a bound-bound transition. The present spectrum peaks around $420 \mathrm{~nm}$ and has no pronounced structure, indicating a bound-free transition. Second, because $\mathrm{H}_{2} \mathrm{O}^{+}$ions are formed almost exclusively in the focus of the laser, ${ }^{34}$ maximum emission should be found if fluorescence is sampled from this region. However, maximum emission is found experimentally if the fluorescence in the region $4 \mathrm{~cm}$ away from the focus is sampled. This is inconsistent with ionic emission. Third, at least four photons are required to form $\mathrm{H}_{2} \mathrm{O}^{+}$with enough energy to explain the

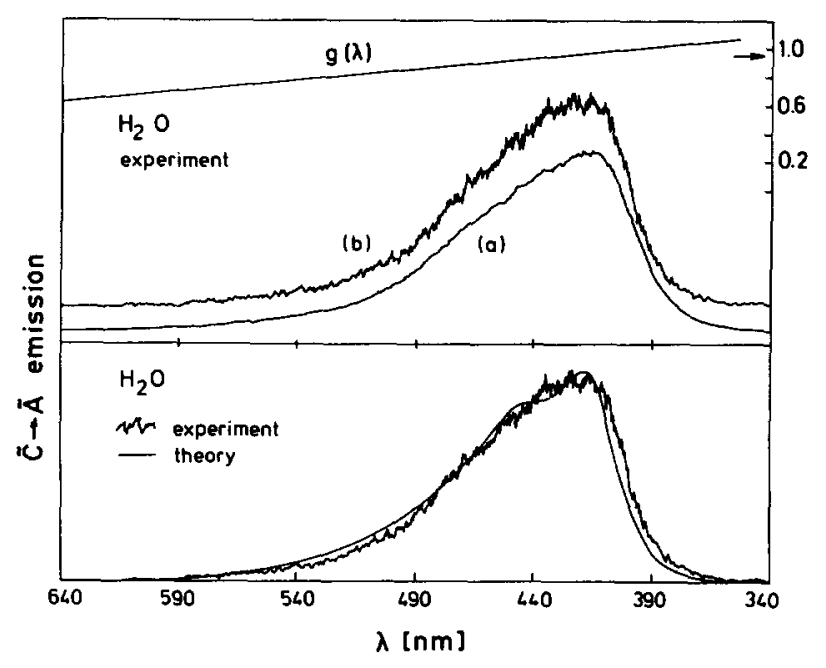

FIG. 3. Upper part: Measured dispersion spectra (linear scale) of $\mathrm{H}_{2} \mathrm{O}$ for two spectral resolutions, $1 \mathrm{~nm}$ (a) and $0.02 \mathrm{~nm}$ (b). $g(\lambda)$ (right scale) is the spectral sensitivity. Lower part: Comparison of the experimental and the theoretical $\widetilde{C} \rightarrow \widetilde{A}$ emission cross section for $\mathrm{H}_{2} \mathrm{O}$ (linear scale). Theory and experiment are normalized at the maximum. 


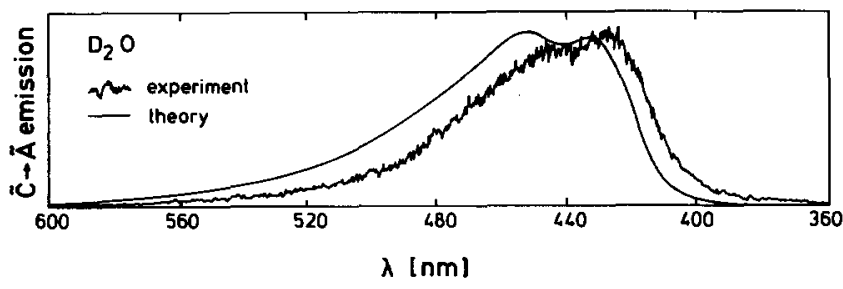

FIG. 4. The same as Fig. 3 but for $\mathrm{D}_{2} \mathrm{O}$.

present emission. However, the observed power dependence is quadratic. The ion formation is almost cubic, ${ }^{34}$ and for electronically excited ions it should at least be more than cubic.

\section{RESULTS}

The upper part of Fig. 3 shows the experimental dispersion spectrum of $\mathrm{H}_{2} \mathrm{O}$, excited with the $\mathrm{KrF}$ laser to the rotational level $2_{02}$ in the $\widetilde{C}$ state. Curve (b) is for a spectral resolution of $0.02 \mathrm{~nm}$, whereas the almost noise-free spectrum (a) is for $\approx 1 \mathrm{~nm}$ resolution. The spectral sensitivity $g(\lambda)$ has been measured in a separate calibration procedure. Curve (a) shows that the emission extends clearly up to almost $600 \mathrm{~nm}$. Curve (b) is added, because it shows a steeper onset of the emission spectrum in the range 390-420 $\mathrm{nm}$. With the lower spectral resolution of curve (a) this onset is smeared out.

In the lower part of Fig. 3 we compare the theoretical and the experimental total emission cross section. The theoretical curve is multiplied by the spectral sensitivity $g(\lambda)$. Theory and experiment are normalized to each other at the maximum. The agreement is as excellent as obtained for the $\widetilde{X} \rightarrow \widetilde{A}$ absorption cross section. ${ }^{14}$ It underlines again the high quality of the calculated $\widetilde{A}$ state potential and also indicates that the assumptions made for the bound wave functions and the transition moment are reliable.

The maximum at around $425 \mathrm{~nm}$ corresponds to a photon energy of $2.9 \mathrm{eV}$. The theoretical spectrum exhibits a slightly indicated undulation at $\sim 440 \mathrm{~nm}$ which is not resolved in the experiment. The same structures are also found in the $\widetilde{X} \rightarrow \widetilde{A}$ absorption cross section ${ }^{14}$ and are clearly resolved there in the measurement of Wang et al. ${ }^{5} \ln$ Ref. 14 we explain them in terms of the individual partial cross sections and the adiabatic potential energy curves in polar coordinates (see also the discussion of Fig. 5 below).

In Fig. 4 we show the corresponding results for $\mathrm{D}_{2} \mathrm{O}$. Excitation has been done on line 17 (see Ref. 34), which simultaneously excites several rotational levels in the $\widetilde{C}$ state. Again the emission extends over a wide range. The experimental spectrum is given with the higher resolution of 0.02 $\mathrm{nm}$ to reproduce the rise as accurately as possible. The theoretical and the experimental spectra are shifted against each other by $\sim 6 \mathrm{~nm}$, measured at the onset at $420 \mathrm{~nm}$. This shift corresponds to an energy of only $0.035 \mathrm{eV}$ which is well below the "theoretical uncertainty" due to the limitations of the model and the estimated accuracy of the calculated $\tilde{A}$ state potential. ${ }^{19}$ Nevertheless, we are surprised that this dis-

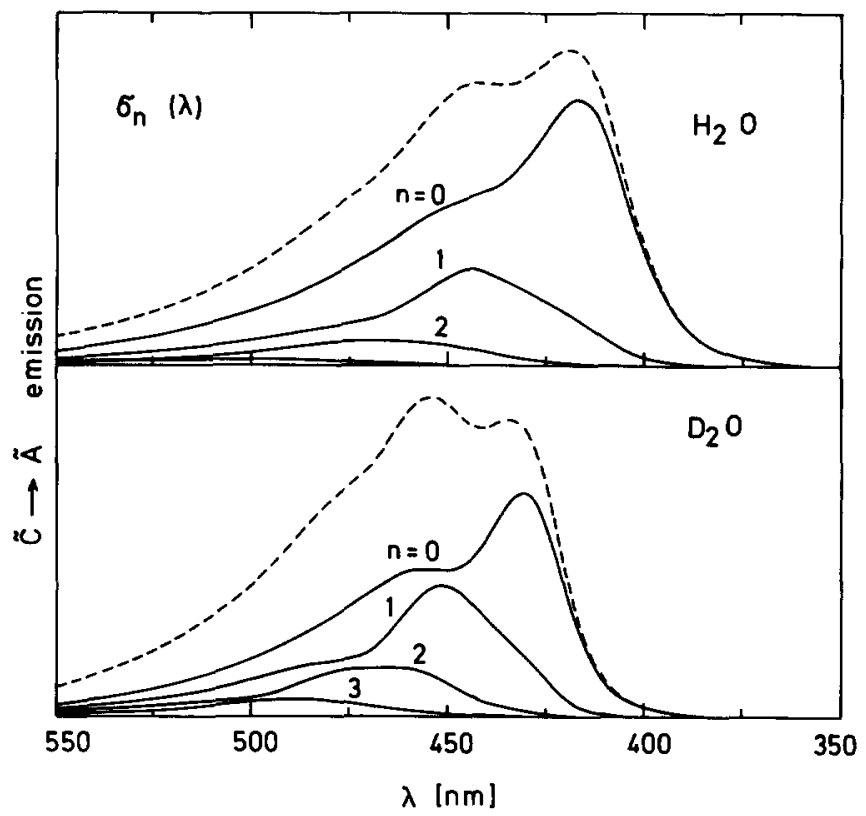

FIG. 5. Wavelength dependence of the partial emission cross sections for vibrational $\mathrm{OH}$ channels $\boldsymbol{n}$ as indicated. The dashed curve is the total cross section, i.e., the sum of all partial cross sections.

crepancy, although small, occurs only for $\mathrm{D}_{2} \mathrm{O}$ but not for $\mathrm{H}_{2} \mathrm{O}$. We have no simple explanation at hand. The undulation superimposed on the maximum is clearly visible both in theory and experiment.

The time-independent theory yields also the partial emission cross sections, i.e., the cross sections for emission of a photon with wavelength $\lambda$ and producing $\mathrm{OH}$ in a specific vibrational state $n$. They are shown in Fig. 5. These cross sections are summed over all final rotational states of $\mathrm{OH}$. As for the $\widetilde{X} \rightarrow \widetilde{A}$ absorption the degree of vibrational excitation becomes larger with increasing excess energy, i.e., with increasing wavelength in the case of emission. However, the $n=0$ cross section is the dominant part over the entire wavelength region. Vibrational excitation is obviously more prominent for $\mathrm{D}_{2} \mathrm{O}$.

The total cross section, also shown in Fig. 5, is simply the sum of all partial cross sections. Figure 5 nicely demonstrates that the weak undulations in the total cross section result from this summation. For example, the peak at $\lambda \sim 435 \mathrm{~nm}$ for $\mathrm{D}_{2} \mathrm{O}$ stems from the $n=0$ cross section while the peak at $455 \mathrm{~nm}$ clearly is due to the $n=1$ cross section. The structures of the individual partial cross sections are analyzed and explained in Refs. 14 and 20.

\section{SUMMARY}

We calculated and measured the $\widetilde{C} \rightarrow \widetilde{A}$ emission spectrum for $\mathrm{H}_{2} \mathrm{O}$ and $\mathrm{D}_{2} \mathrm{O}$. The calculations are based on a rigorous quantal treatment of the dissociation dynamics on the repulsive $\widetilde{A}$ state potential energy surface which has been calculated with quantum chemical methods. The weakest points of the model are the description of the bond wave functions in the $\widetilde{C}$ state and the assumption of a constant $\widetilde{C}-$ $\tilde{A}$ transition dipole function. The very good agreement 
between theory and experiment, however, indicates that these assumptions are reasonably realistic. The slight shift of $6 \mathrm{~nm}$ found for $\mathrm{D}_{2} \mathrm{O}$ corresponds to only $0.035 \mathrm{eV}$ which is less than $2 \%$ of the photon energy or the excess energy at 440 nm.

Process (3) in Fig. 2 is -in principle-rather complicated. It consists of a one (or two) photon excitation from $\widetilde{X}$ to $\widetilde{C}$, strong predissociation via the $\widetilde{B}$ state, and emission from the $\widetilde{C}$ state to the repulsive $\widetilde{A}$ state. A rigorous quantal theory therefore should include simultaneously all three electronic states. In our model we ignore predissociation and assume that the quasibound state is a real bound state. The excellent agreement with the measurements indicates that this assumption is reliable. However, in the present work we considered only the wavelength dependence of the emission spectra. If we would attempt to calculate also the absolute values we would have to include the nonadiabatic coupling between $\widetilde{C}$ and $\widetilde{B}$ which determines the predissociation rate and therefore the lifetime in the $\widetilde{C}$ state.

\section{ACKNOWLEDGMENTS}

R.S. would like to acknowledge stimulating discussions with Professor Simons and Dr. Ashfold in the early stage of this project. Financial support of the Deutsche Forschungsgemeinschaft is gratefully acknowledged (V.E. and R.S.).

${ }^{1}$ K. Watanabe and M. Zelikoff, J. Opt. Soc. Am. 41, 753 (1953).

${ }^{2}$ S. Bell, J. Mol. Spectrosc. 16, 205 (1964).

${ }^{3}$ T. Carrington, J. Chem. Phys. 41, 2012 (1964).

${ }^{4}$ J. W. C. Johns, Can. J. Phys. 49, 944 (1971).

${ }^{5}$ H.-T. Wang, W. S. Felps, and S. P. McGlynn, J. Chem. Phys. 67, 2614 (1977).

${ }^{6}$ M. N. R. Ashfold, J. M. Bayley, and R. N. Dixon, Chem. Phys. 84, 35 (1984).

7J. P. Simons, A. J. Smith, and R. N. Dixon, J. Chem. Soc. Faraday Trans. 2 80, 1489 (1984).

${ }^{8}$ P. Andresen, G. S. Ondrey, B. Titze, and E. W. Rothe, J. Chem. Phys. 80, 2548 (1984).

${ }^{9}$ H. J. Krautwald, L. Schnieder, K. H. Welge, and M. N. R. Ashfold, Fara- day Discuss. Chem. Soc. 82, paper 7 (1986).

${ }^{10}$ F. Flouquet and J. A. Horsley, J. Chem. Phys. 60, 3767 (1974).

${ }^{11}$ R. E. Howard, A. D. McLean, and W. A. Lester, Jr., J. Chem. Phys. 71, 2412 (1979).

${ }^{12}$ G. Theodorakopoulos, I. D. Petsalakis, and R. J. Buenker, Chem. Phys. 96, 217 (1985).

${ }^{13}$ R. Segev and M. Shapiro, J. Chem. Phys. 77, 5604 (1982).

${ }^{14} \mathrm{~V}$. Engel, R. Schinke, and V. Staemmler, Chem. Phys. Lett. 130, 413 (1986).

${ }^{15}$ M. B. Robin, Higher Excited States of Polyatomic Molecules (Academic, New York, 1974); H. Okabe, Photochemistry of Small Molecules (Wiley, New York, 1978).

${ }^{16} \mathrm{~S}$. Theodorakopoulos, I. D. Petsalakis, R. J. Buenker, and S. D. Peyerimhoff, Chem. Phys. Lett. 105, 253 (1984).

${ }^{17}$ P. Andresen, V. Beushausen, D. Häusler, and H. W. Lülf, J. Chem. Phys. 83, 1429 (1985).

${ }^{18}$ R. Schinke, V. Engel, P. Andresen, D. Häusler, and G. G. Balint-Kurti, Phys. Rev. Lett. 55, 1180 (1985).

${ }^{19}$ V. Staemmler and A. Palma, Chem. Phys. 93, 63 (1985).

${ }^{20}$ V. Engel, R. Schinke, and V. Staemmler, J. Chem. Phys. (in press).

${ }^{21} \mathbf{P}$. Andresen and R. Schinke, in Molecular Photodissaciation Dynamics, edited by J. E. Baggott and M. N. R. Ashfold (Royal Society of Chemistry, London, 1987).

${ }^{22}$ J. P. Simons, J. Phys. Chem. 88, 1287 (1984).

${ }^{23}$ A. Hodgson, J. P. Simons, A. J. Smith, and R. N. Dixon, in Photophysics and Photochemistry above $6 \mathrm{eV}$, edited by F. Lahmani (Elsevier, Amsterdam, 1985).

${ }^{24}$ E. Segev and M. Shapiro, J. Chem. Phys. 73, 2001 (1980).

${ }^{25}$ I. P. Vinogradov and F. I. Vilesov, Opt. Spectrosc. 40, 32 (1976).

${ }^{26}$ R.N. Dixon, Mol. Phys. 54, 333 (1985).

${ }^{27}$ M. P. Docker, A. Hodgson, and J. P. Simons, Mol. Phys. 57, 129 (1986).

${ }^{28}$ M. Shapiro and R. Bersohn, Annu. Rev. Phys. Chem. 33, 409 (1982); M. Shapiro and G.G. Balint-Kurti, in Photodissociation and Photoionization, edited by K. P. Lawley (Wiley, New York, 1985), p. 403.

${ }^{29}$ D. Secrest, J. Chem. Phys. 62, 710 (1975); L.W. Hunter, ibid. 62, 2855 (1975).

${ }^{30}$ J. Manz, Comments At. Mol. Phys. 17, 91 (1985).

${ }^{31}$ R. Schinke, V. Engel, and V. Staemmler, J. Chem. Phys. 83, 4522 (1985).

${ }^{32}$ K. S. Sorbie and J. N. Murrell, Mol. Phys. 29, 1387 (1975); 31, 905 (1976).

${ }^{33}$ V. Engel, R. Schinke, and V. Staemmler, Chem. Phys. Lett. 116, 165 (1985).

${ }^{34}$ G. Meijer, J.J. terMeulen, P. Andresen, and A. Bath, J. Chem. Phys. 85, 6914 (1986).

${ }^{35}$ R. Derai, S. Fenistein, M. Gerard-Ain, T.R. Govers, R. Marx, G. Mauclaire, C. Z. Profous, and C. Sourisseau, Chem. Phys. 44, 65 (1979). 